\title{
Case report of individual with cutaneous immunodeficiency and novel Ip36 duplication
}

\author{
This article was published in the following Dove Press journal: \\ The Application of Clinical Genetics \\ 13 January 2016 \\ Number of times this article has been viewed
}

\author{
Alyn D Hatter' \\ David C Soler ${ }^{2,3}$ \\ Christine Curtis ${ }^{4}$ \\ Kevin D Cooper ${ }^{1,2,3,5}$ \\ Thomas S McCormick 2,3 \\ 'University Hospitals Case Medical \\ Center, ${ }^{2}$ Department of Dermatology, \\ ${ }^{3}$ The Murdough Family Center for \\ Psoriasis, Case Western Reserve \\ University, ${ }^{4}$ Cleveland Department \\ of Pathology and Center for Human \\ Genetics Laboratory, ${ }^{5}$ VA Medical \\ Center, Cleveland, OH, USA
}

Introduction: Crusted or Norwegian scabies is an infectious skin dermatopathology usually associated with an underlying immunodeficiency condition. It is caused when the mite Sarcoptes scabiei infects the skin, and the immune system is unable to control its spread, leading to a massive hyperinfestation with a simultaneous inflammatory and hyperkeratotic reaction. This is the first report of a novel 1p36 duplication associated with a recurrent infection of crusted scabies.

Case report: We describe a 34-year-old patient with a cutaneous immunodeficiency characterized by recurrent crusted scabies infestation, diffuse tinea, and recurrent staphylococcal cellulitis, who we suspected had an undiagnosed syndrome. The patient also suffered from mental retardation, renal failure, and premature senescence. A cytogenetic fluorescence in situ hybridization analysis revealed a $9.34 \mathrm{Mb}$ duplication within the short (p) arm of chromosome 1 , precisely from $1 \mathrm{p} 36.11$ to $1 \mathrm{p} 36.21$, with an adjacent $193 \mathrm{~kb}$ copy gain entirely within $1 \mathrm{p} 36.11$. In addition, chromosome 4 had a $906 \mathrm{~kb}$ gain in $4 \mathrm{p} 16.1$ and chromosome 9 had a $81 \mathrm{~kb}$ copy gain in 9p24.3. Over 100 genes localized within these duplicated regions. Gene expression array revealed 82 genes whose expression changed $>1.5$-fold compared to a healthy age-matched skin control, but among them only the lipolytic enzyme arylacetamide deacetylase-like 3 was found within the duplicated $1 \mathrm{p} 36$ region of chromosome 1 .

Discussion: Although genetic duplications in the 1 p36 region have been previously described, our report describes a novel duplicative variant within the 1 p36 region. The patient did not have a past history of immunosuppression but was afflicted by a recurrent case of crusted scabies, raising the possibility that the recurrent infection was associated with the $1 \mathrm{p} 36$ genetic duplication.

Conclusion: To our knowledge, the specific duplicated sequence between 1p36.11 and p36.21 found in our patient has never been previously reported. We reviewed and compared the clinical, genotyping, and gene microarray results of our patient in order to characterize this novel 1p36 duplication syndrome, which might have contributed to the recurrent scabies infection in this patient.

Keywords: immunodeficiency, crusted scabies, cutaneous immunodeficiency, 1p36 duplication

\section{Introduction}

Crusted scabies is a rare, severe form of skin infection caused by the mite Sarcoptes scabiei var. homini. It is often associated with the elderly or with people who have a compromised immune system such as with HIV infection or in patients receiving immunosuppressive treatments. However, very few cases have been described of crusted scabies affecting young and relatively immune-competent patients.

Herein, we report a case of a patient with a cutaneous idiopathic immunodeficiency characterized by recurrent crusted scabies infestation as well as chronic fungal and bacterial infection. We suspected an undiagnosed syndrome. We detail her phenotype
Correspondence: David C Soler Department of Dermatology, Case Western Reserve University, BRB Building Room 547B, 2109 Adelbert Road, Cleveland, OH 44106, USA

Tel +l $216368 \mid 262$

$\mathrm{Fax}+1216368.0212$

Email david.soler@case.edu (c) (i) (9) 2016 Hatter et al. This work is published by Dove Medical Press Limited, and licensed under Creative Commons Attribution - Non Commercial (unported, v3.0) License. The full terms of the License are available at http://creativecommons.org/licenses/by-nc/3.0/. Non-commercial uses of the work are permitted without any further permission for the license are administered by Dove Medical Press Limited. Information on how to request permission may be found at: http://www.dovepress.com/permissions.php 
and clinical history, and report our findings from cytogenetic and cutaneous gene microarray analysis.

\section{Case report}

A 34-year-old Caucasian woman was referred to the department of dermatology for evaluation of recurrent and diffuse tinea corporis and recalcitrant crusted scabies. All studies of human subjects were approved by the Institutional Review Board of University Hospitals Case Medical Center (Cleveland, OH, USA) and the patient provided written informed consent. Earlier treatments included topical ketoconazole $2 \%$ cream, oxiconazole nitrate $1 \%$ cream, and permethrin $5 \%$ cream. The patient reported that her skin problems had been intermittent over the preceding 3 years. The medical history of this patient revealed end-stage renal disease requiring hemodialysis, obesity, non-insulin-dependent diabetes, hypertension, recurrent cellulitis, and methicillin-resistant Staphylococcus aureus bacteremia. Recently, prior to our consultation, she was diagnosed with bilateral cataracts and mild sensorineural hearing loss in one ear. Diabetes and hypertension were coincidentally diagnosed with nephrotic syndrome at age 29 during an acute illness, and she subsequently began hemodialysis. The patient had frequent admissions thereafter for dialysis catheter site cellulitis and methicillin-resistant S. aureus bacteremia secondary to catheter line infections. The patient has lived with her maternal grandmother since birth and was reportedly in good health until age 29 with no evidence of childhood infections other than the infrequent "cold". The patient had no known siblings and the father was unknown to the family; the mother reportedly suffered from chronic pain related to an injury and died from a prescription narcotic overdose at age 29. There was no evidence of birth defects or immunodeficiency in the maternal family.

Physical examination revealed a woman appearing much older than her chronological age would suggest. The patient exhibited coarse facial features with a prominent brow ridge, midface hypoplasia, prognathia, and slight down slanting of palpebral fissures (Figure 1). The skin examination was significant for thick, hyperkeratotic, white, and flaking plaques on the palms, soles, digits, and under the distal nails (Figure 2). Moist erosions and bright erythema along many of the proximal nail folds were noted. The nail plates appeared normal except distal onycholysis overlying the plaques. There were crusted papules and erosions diffusely scattered on the face, ears, trunk, and extremities consistent with excoriations. There was no evidence of active tinea at the time of the patient visit. Earlier mineral oil scrapings had revealed multiple live mites and exam was consistent with crusted scabies. A fungal
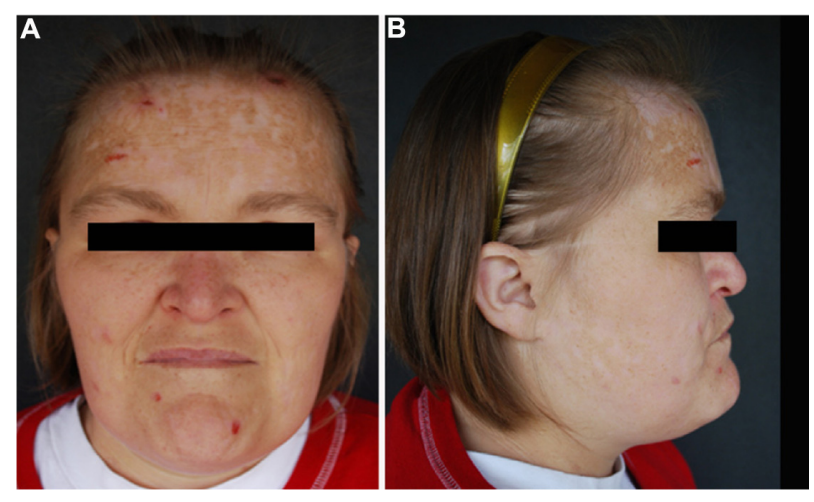

Figure I Our patient demonstrates coarse facial features (A), hypoplastic midface and prognathia (B).

culture from the nail fold grew unidentified, non-Candida yeast. It was recommended to the referring dermatologist that the patient continues topical antifungal treatment as needed and to begin oral ivermectin $0.2 \mathrm{mg} / \mathrm{kg}$ daily on days $1,2,8$, $9,15,22$, and $29 .{ }^{1}$ An undiagnosed immune syndrome with cutaneous-specific immunodeficiency was suspected, so we referred the patient to medical genetics for evaluation. The laboratory results and hospital admission notes from her community physician were reviewed. Importantly, the patient was HIV negative, had a normal serum protein electrophoresis interpretation, and was negative for antinuclear antibodies. Electrolyte panels were consistent with renal failure and routine dialysis, but no renal biopsy had been performed. Although the white blood cell count would fluctuate between admissions, the patient's lymphocyte count was consistently within normal limits. The scabies infection resolved after 3 months using routine maintenance doses of ivermectin. The tinea corporis infection resolved using the daily application of topical antifungal creams.

\section{Results}

Cytogenetic analysis revealed a female karyotype with a duplication within the short (p) arm of chromosome 1. Duplication breakpoints were assigned to G-band regions $1 \mathrm{p} 36.11$ and $1 \mathrm{p} 36.21$ [dup(1)(p36.11p36.21)] (Figure 3A).

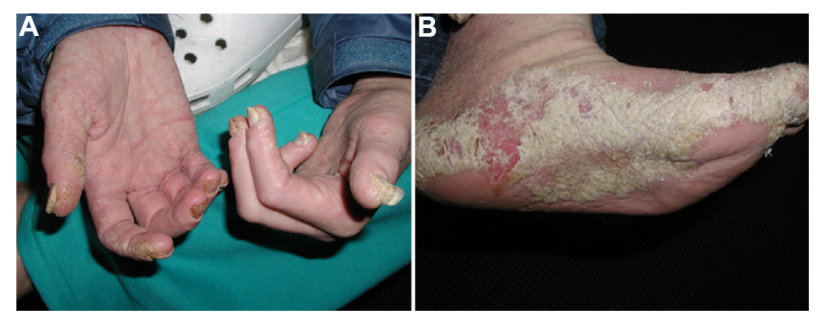

Figure 2 Our patient had hyperkeratotic white plaques on her soles, hands, and periungual digits (A and $\mathbf{B}$ ); manifestations of crusted scabies (B). 
Fluorescence in situ hybridization (FISH) was performed on metaphase cells from the sample, using two bacterial artificial chromosome probes (RP11-139H5 and -430L17) which localize to G-band regions $1 \mathrm{p} 36.12$ and $1 \mathrm{p} 36.13$, respectively (both of which lie within the duplicated segment). Each probe hybridized to two sites along the duplicated 1p arm, confirming the duplication of $1 \mathrm{p}$ material (Figure 3B). The relative positioning of the two probes suggests that the duplicated segment is adjacent to the original chromosomal segment in an inverted orientation. OligoArray analysis, using Signature Select OS 105K v1.1 array (Signature Genomics, Spokane, WA, USA), demonstrated an approximately 9.3 $\mathrm{Mb}$ copy gain within chromosome bands 1p36.11-p36.21. This gain is adjacent to an approximately $62 \mathrm{~kb}$ segment of a normal copy number within $1 \mathrm{p} 36.11$, followed by another gain of $193 \mathrm{~kb}$ within $1 \mathrm{p} 36.11$.

In addition, the array identified two smaller regions of copy gain - a $906 \mathrm{~kb}$ gain in chromosome $44 \mathrm{p} 16.1$ and an $81 \mathrm{~kb}$ copy gain in chromosome 9 9p24.3. The 4p16.1 region includes approximately seven genes and is recognized as a region of significant copy variation and segmental duplication. ${ }^{2}$ There are no FISH probes within this region. ${ }^{3}$ The small 9p24.3 region contains the $5^{\prime}$ portion of the DOCK 8 gene and the $3^{\prime}$ portion of the C9orf66 gene. The copy number variation has been identified for this region ${ }^{3}$. The FISH analysis failed to confirm a duplication of this

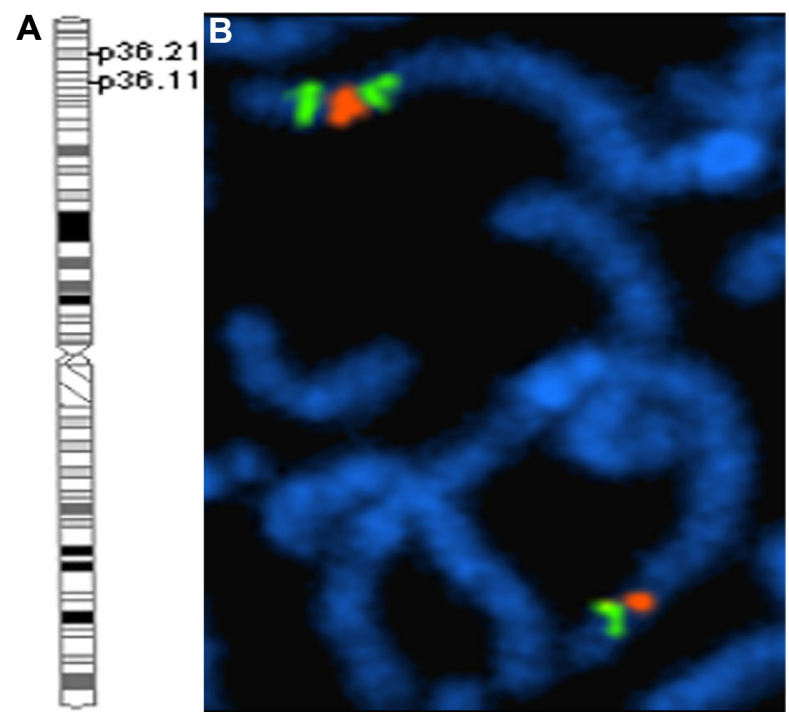

Figure 3 Cytogenetic and FISH analysis.

Notes: (A) Ideogram of G-banding pattern for normal chromosome I. The region included in the patient's duplicated segment is indicated by its p36.1I and p36.2I breakpoints. (B) Partial G-banded karyotype of the patient's chromosomes I. The duplicated chromosome $I$ is on the left and the normal chromosome $I$ is on the right. The green bands identify the p36.II duplication breakpoint; the red bands identify the $\mathrm{p} 36.21$ duplication breakpoint.

Abbreviation: $\mathrm{FISH}$, fluorescence in situ hybridization. region, but a simultaneous hybridized control probe showed the normal signal number and pattern. It is therefore likely that the size of the duplication is below the technical detection limit for FISH.

Fragile $\mathrm{X}$ testing was negative and a plasma amino acid profile was within normal limits.

When using 1.5-fold as a threshold for measuring differences in levels of expression, we discovered 82 genes with $>1.5$-fold difference. Of the genes within the duplication, only one, arylacetamide deacetylase-like 3 (AADACL3, HGNC: 32037), was within the known duplicated region on chromosome 1 . The $A A D A C L 3$ belongs to a lipolytic enzyme family with unknown function or significance in the skin. Thus, although the duplicated regions contained more than 100 genes, these do not appear to have been upregulated at the mRNA level in unaffected tissue.

\section{Discussion}

We present our observations of a 34-year-old patient with cutaneous immunodeficiency, renal failure, and premature senescence ultimately diagnosed with an 1p36 duplication. The duplications within the 1 p36 region have been previously reported in the patients with variable clinical phenotypes including, but not limited to: atrial septal defects, delayed gross motor development, mental retardation, microcephaly, craniosynostosis, minor facial anomalies, patent ductus arteriosus, transient hypogammaglobulinemia, and seizures. ${ }^{4,5}$ However, the specific duplicated sequences between 1p36.11-p36.21, 4p16.1, and 9p24.3 found in our patient have not been reported elsewhere. The comparisons of gene expression in skin samples between our patient and a control subject revealed 82 genes with at least 1.5 -fold difference in levels of expression. Of these, only AADACL3 is located within our patient's known chromosomal duplications, but the function of this gene and its significance in skin immunity is currently unknown. Phenotyping multiple patients and running microarray comparisons would increase the characterization of the 1p36 duplication genotype. However, the uniqueness of this patient's duplicated 1p36.11-p36.21 as well as 4p16.1 and 9p24.3 regions posed a challenge and to date we have been unable to find any similar patients to study.

Finally, although skin immunodeficiency may be a result of the 1 p36 duplication, caution is warranted regarding the conclusion based on only one patient observed with this genotype. The large number of genes within the duplicated regions and the limited patient availability precluded any further functional assessment in order to find a causative 
gene or set of genes within the duplicated regions responsible for the patient's cutaneous immunodeficiency. A possible research direction could be undertaken by coculturing the patient's keratinocytes with S. scabiei and quantifying by enzyme-linked immunosorbent assay the production of IL-21 and other relevant keratinocyte-derived cytokines that may be upregulated and potentially responsible for inhibiting this skin infection through recruitment of cellular immune mediators.

\section{Conclusion}

The recurrent crusted scabies infection in our patient diagnosed with a rare 1 p36 duplication represents a novel phenotypic manifestation of the 1 p36 duplication syndrome. To our knowledge, this is the first report of a young female patient with the 1 p36 duplication associated with a recurrent crusted scabies infection of the skin. This case serves to further describe a previously unknown type of an 1p36 duplication described in the context of a recurrent skin infection normally associated with the patients suffering from immunosuppression.

\section{Acknowledgments}

This study was supported in part by the National Institutes of Health (P30AR39750), Department of Dermatology Resident and Education Fund. The funding agency had no involvement in study design, data collection, data analysis, manuscript preparation, and/or publication decisions.

\section{Disclosure}

The authors report no conflicts of interest in this work.

\section{References}

1. Currie BJ, McCarthy JS. Permethrin and ivermectin for scabies. N Engl J Med. 2010;362:717-725.

2. Redon R, Ishikawa S, Fitch KR, et al. Global variation in copy number in the human genome. Nature. 2006;444:444-454.

3. Zogopoulos G, Ha KC, Naqib F, et al. Germ-line DNA copy number variation frequencies in a large North American population. Hum Genet. 2007;122:345-353.

4. Chen E, Obolensky E, Rauen KA, et al. Cytogenetic and array CGH characterization of de novo $1 \mathrm{p} 36$ duplications and deletion in a patient with congenital cataracts, hearing loss, choanal atresia, and mental retardation. Am J Med Genet A. 2008;146A:2785-2790.

5. Brandigi E, Molinaro F, Bulotta AL, et al. Chromosome 18q-Syndrome and $1 \mathrm{p}$ terminal duplication in a patient with bilateral vesico-ureteral reflux: case report and literature revision. Ital J Pediatr. 2013;39:6.
The Application of Clinical Genetics

\section{Publish your work in this journal}

The Application of Clinical Genetics is an international, peer-reviewed open access journal that welcomes laboratory and clinical findings in the field of human genetics. Specific topics include: Population genetics; Functional genetics; Natural history of genetic disease; Management of genetic disease; Mechanisms of genetic disease; Counseling and ethical

\section{Dovepress}

issues; Animal models; Pharmacogenetics; Prenatal diagnosis; Dysmorphology. The manuscript management system is completely online and includes a very quick and fair peer-review system, which is all easy to use. Visit http://www.dovepress.com/testimonials.php to read real quotes from published authors. 